\title{
The epiconus syndrome presenting with radicular-type neurological features
}

\author{
Yasumitsu Toribatake ${ }^{1}$, Hisatoshi Baba ${ }^{2}$, Norio Kawahara ${ }^{1}$, Katsunori Mizuno ${ }^{1}$ and Katsuro Tomita ${ }^{1}$ \\ ${ }^{1}$ Department of Orthopaedic Surgery, School of Medicine, Kanazawa University, 13-1 Takaramachi, Kanazawa 920; \\ ${ }^{2}$ Department of Orthopaedic Surgery, Fukui Medical School, Shimoaizuki 23, Matsuoka, Fukui 910-11, Japan
}

\begin{abstract}
We report 15 patients with an epiconus syndrome presenting with radicular-type clinical features involving the lower extremities and identify the vertebral level at which the spinal cord terminated. All patients, consisting of 10 men and five women, aged 37 to 71 years, presented with unilateral or bilateral leg muscle atrophy as well as sensory deficit. Unilateral or bilateral reduction or absence of deep tendon reflexes were present in 13 patients, while pathological reflexes were seen in three cases and bladder dysfunction in 10. Prior to presentation, five patients with the epiconus syndrome were mistakingly diagnosed as having different spinal diseases, which were treated surgically. The level of epiconus compromise detected on radiological examination ranged from the lower level of the T10 vertebra to T12L1 intervertebral disc level, but most frequently at T11-12 disc level. The level of termination of the spinal cord was between T12 and L1-2 disc level, but most frequently at the middle portion of the L1 vertebra, which was on average $1.6 \pm 0.4$ (mean \pm standard deviation) vertebrae distal to the epiconus lesions. The presence of different neurological features related to epiconus compromise should be carefully examined with reference to the level of termination of the spinal cord.
\end{abstract}

Keywords: thoracolumbar spine; epiconus; termination of the spinal cord; neurological symptomatology; neuroimaging

\section{Introduction}

Spinal cord compromise at the thoracolumbar junction may infrequently present with a complex neurological symptomatology. The neuroanatomical complexity of the spinal cord at this level is associated with a wide spectrum of clinical manifestations particularly when the region is involved by different lesions. A lesion of the epiconus (L4 to S1 spinal cord segments) can produce, as weakness develops, a flaccid type paralysis with signs of lower motor neuron involvement. ${ }^{1,2}$ When the lesion involves the spinal cord above the epiconus, spastic paraesis as a sign of upper motor neuron dysfunction may also occur. Similarly, when the lesion involves the cord distal to the epiconus, bladder dysfunction, as a sign of conus medullaris compromise, and the cauda equina syndrome is usually evident on clinical presentation. Accordingly, when suspecting a lesion in the epiconus segment, one should be careful in evaluating the results of neurological and neuroimaging examinations. The epiconus syndrome, particularly radicular-type, can present with symptoms and signs that mimic those of lumbar nerve root involvement and/or some forms of peripheral nerve entrapment.

Correspondence: Y Toribatake
The current communication provides a description of the clinical spectrum of the epiconus syndrome, particularly in those patients presenting primarily with radicular-type leg symptoms. The results were stratified according to the neuroanatomical location of spinal cord termination.

\section{Patients and methods}

Patients and clinical assessment

Of 135 patients with neurological compromise who were treated for spine and spinal cord diseases at thoracolumbar junction, 15 patients ( 10 men and five women) were found to have the epiconus syndrome and showed radicular-type clinical symptoms and signs affecting the lower extremities. The age at operation ranged from 37 to 71 years (average, 56 years), and the syndrome was found to be caused by different forms of lesions at that stage (Table 1). The duration between the onset of symptoms and the time of diagnosis of the epiconus syndrome ranged from 0 to 168 months (average, 32 months). Herniated lumbosacral intervertebral disc and idiopathic peroneal nerve palsy were the commonest provisional diagnoses before presentation. Prior to admission, five patients with the epiconus syndrome (Cases 2, 3, 4, 5 and 


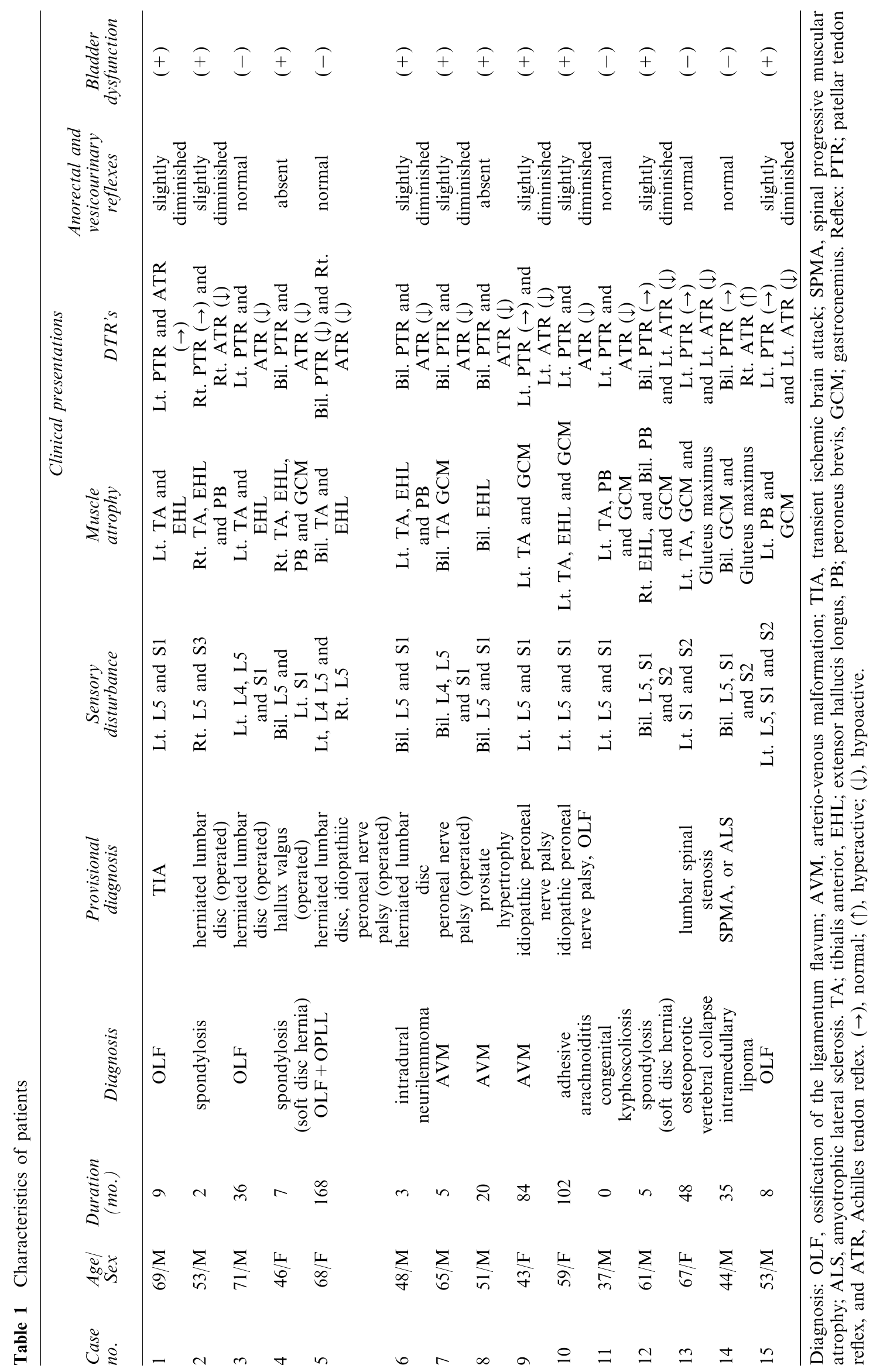


7) were mistakingly diagnosed as having different spinal diseases, which were treated surgically.

We assessed the neurological status of each patient, particularly the level of spinal cord involvement and the termination of the spinal cord. The present series of patients presented with a unilateral or a bilateral radicular-type disturbance of sensory function of the lower extremities. Urodymamic studies were indicated when the patient reported urinary problems or discomfort. In patients with lesions spanning over multiple vertebral levels, we also recorded epidural spinal cord evoked potentials during surgery to determine the level most responsible for the neurological complication, as we have reported recently.,

\section{Radiological examination}

Radiological investigations included computed tomography myelography and/or magnetic resonance imaging (MRI). Following the detection of the epiconus lesion, we carefully studied the level of termination of the spinal cord and continuation as the cauda equinae and the film terminale. The vertebral level of the epiconus involvement and the level of termination of the spinal cord were divided into four segments: the level corresponding to the upper third of the vertebral body (denoted as ' $U$ ' on radiological assessment shown in Table 2); middle third (as 'M'); lower third (as ' $L$ '); or the intervertebral disc level (as 'D'). Such division allowed us to study the anatomical relationship between the epiconus lesion and the level of termination of the spinal cord in each individual.

\section{Results}

Clinical features

Table 1 summarises the anthropometric and clinical characteristics of our patients. Clinical features indicative of unilateral leg involvement were present in nine cases, and of bilateral involvement in six patients. Several patients (Cases 2, 4, 10 and 15; Table 1) complained of a radicular-type pain radiating to the involved leg areas or reported an abnormal tingling sensation of the feet as well as perineal area (Case 12).

The most frequently found sensory deficits included a unilateral or bilateral disturbance of sensation in the L5 dermatome (14/15 cases) and an S1 sensory deficit (13/15 cases). Symptoms related to the S2-S4 dermatomes, suggestive of coexisting involvement of the conus medullaris, were present in five patients. Disturbances of the vibration sensation were present in all of the patients except Case 6, 12 and 14 .

Atrophy of the lower leg muscles was present in close association with sensory dysfunction. Thus, atrophy of the gastrocnemius muscle was evident in nine patients, while weakness of the tibialis anterior was detected in seven, unilaterally and in two patients bilaterally. Diminished or absent deep tendon reflexes on one or two sides was present in 13 of the patients. One patient presented with a hyperactive Achilles tendon reflex on one side but the patellar tendon reflex was normal on both sides (Case 14). The Babinski's reflex was present (positive) in three patients (Cases 1 , 10 and 14). Anorectal and vesicourinary reflexes were diminished or absent in 10 patients, and 10 had bladder dysfunction based on the symptoms or detected on urodynamic tests.

Level of lesions and termination of the spinal cord The vertebral levels at which the epiconus was involved are shown in Table 2. The level varied widely, ranging from the lower third level (L) of T10 vertebra to the intervertebral disc level (D) at T12-L1. The lesion showed a topographic distribution of two vertebrae and two intervertebral discs. The intervertebral disc

Table 2 Radiologic data and treatment

\begin{tabular}{|c|c|c|c|c|}
\hline Case No. & $\begin{array}{l}\text { Vertebral level } \\
\text { of the lesion }\end{array}$ & $\begin{array}{l}\text { Vertebral level of termin- } \\
\text { ation of the spinal cord }\end{array}$ & $\begin{array}{l}\text { Distance between the lesion } \\
\text { and termination of the cord } \dagger\end{array}$ & $\begin{array}{c}\text { Surgery for the } \\
\text { lesion }\end{array}$ \\
\hline 1 & T10-11 (D) & $\mathrm{T} 12$ (L) & 1.75 & laminectomy \\
\hline 2 & T12-L1 (D) & L1-2 (D) & 1.0 & anterior decompression \\
\hline 3 & T11-12 (D) & L1 (M) & 1.5 & laminectomy \\
\hline 4 & T11-12 (D) & L1 (M) & 1.5 & anterior decompression \\
\hline 5 & T11-12 (D) & L1 (U) & 1.25 & laminectomy \\
\hline 6 & T11-12 (D) & L1 (M) & 1.5 & resection \\
\hline 7 & T11-12 (D) & L1 (M) & 1.5 & resection (coagulation) \\
\hline 8 & T10 (L) & T12 (L) & 2.0 & resection (coagulation) \\
\hline 9 & T11-12 (D) & L1 (L) & 1.75 & resection \\
\hline 10 & $\mathrm{~T} 12(\mathrm{M})$ & L1-2 (D) & 1.5 & resection (and neurolysis) \\
\hline 11 & T12 (U) & L1-2 (D) & 1.75 & anterior decompression \\
\hline 12 & T11-12 (D) & L1 (M) & 1.5 & anterior decompression \\
\hline 13 & T12-L1 (D) & L1-2 (D) & 1.0 & posterior decompression \\
\hline 14 & $\mathrm{~T} 12(\mathrm{M})$ & L1-2 (D) & 1.5 & paliative resection \\
\hline 15 & T11-12 (D) & L1 (L) & 2.25 & laminectomy \\
\hline
\end{tabular}

*: U, upper; M, middle; L, lower; and D, intervertebral disc level, $\dagger$ : distance $=$ [each score] $\mathrm{X}$ [one vertebral height]. 
level (D) at T11-T12 was the most frequent site of epiconus compromise (seven cases).

Termination of the spinal cord occurred between the lower third level (L) of T12 and intervertebral disc level (D) at L1-2. The level of termination of the spinal cord was at the middle third portion (M) of L1 in five patients while in another five patients, this was at the L1-2 intervertebral disc level (D). The distance between levels where the epiconus was involved and termination of the spinal cord, ranged from 1.0 to 2.5 vertebrae, with an average distance of $1.6 \pm 0.4$ vertebrae (mean \pm standard deviation).

\section{Case presentations}

\section{Case 6}

The chief complaint of this 48-year-old man was a 3month history of gait disturbance. He was unable to lift his left foot, and developed numbness of the lateral aspect of the calf. This was followed by slight difficulty in voluntary micturition. On presentation, sensory disturbances of the L5 and S1 dermatomes was evident bilaterally and vibration sensation in the lower leg was diminished on both sides. The strength of the left tibialis anterior and extensor hallucis muscles was [2-3/5], while that of the left peroneus brevis and gastrocnemius muscles was [4/5]. The patellar and Achilles tendon reflexes were hypoactive bilaterally. There were also clinical features of bladder dysfunction including urinary retention and dysuria.

Multiple intradural and extramedullary tumours were evident on CT and MRI scans between T11 and L2 vertebral levels, and the level of termination of the spinal cord was at the middle portion of L1 (Figure 1a-c). Multiple intradural extramedullary neurilemmomas were removed using a microsurgical technique. The tumour at T11-12 (D) was 1.5 vertebra cephalad to the level where the spinal cord terminated and was determined to be responsible for the epiconus syndrome on intraoperative spinal cord evoked potential study.

\section{Case 10}

A 59-year-old woman developed progressive weakness of her left foot 8 years before presentation. On examination, she had a left foot drop with significant atrophy of the left tibialis anterior (strength, 1-2/5), extensor hallucis longus (4/5) and gastrocnemius muscles $(3 / 5)$. Sensory disturbances were present in the left L5 and S1 dermatomes, and diminished vibration sensation was detected on the lower leg bilaterally. Deep tendon reflexes were slightly hypoactive on the left, but Babinski's reflex was positive on the same side. She had bladder and bowel dysfunction.

Radiological examination showed ossification of the posterior longitudinal ligament and the ligamentum flavum at T8-T11, with the lesion at T9-10 level being the most significant one (Figure $2 \mathrm{a}$ ). In addition, an intradural lesion at the middle portion of the T12 vertebra suggestive of arachnoiditis was visible on MRIs (Figure 2b). The lesion was determined to be responsible for the epiconus syndrome, based on intraoperative spinal evoked potential recording (Figure 2c).

\section{Case 13}

This was a 67-year-old woman who had been treated with medications for a long period for osteoporosis. About 6 years before presentation, she sustained a compression fracture of L1 vertebra without neurological compromise. However, 1 year later, she noticed progressive weakness of her left calf and foot muscles as well as slight numbness of the sole of the left foot. A tentative diagnosis of idiopathic peroneal nerve palsy was made. On presentation at our clinic, she was ambulatory aided with a cane because of the left foot drop. Tests for neurological function of the upper extremity were normal but gait was slightly flaccid. Romberg's sign was negative. The strength of the left tibialis anterior and extensor hallucis muscles was [3/5], while that of the left gastrocnemius muscle was $\left[4^{+} / 5\right]$. She also had atrophy of the left gluteus maximus muscle. There was a slighly sensory deficit at S1 and S2 dermatomes on the left leg. The patellar tendon reflex was normal on both sides, but Achilles tendon reflex was hypoactive on both sides, particularly on the right leg. She had a slight difficulty in voluntary micturition but the results of a urodynamic study were within the normal limit.

Cervical MRIs showed multiple, asymptomatic, compressions extending from $\mathrm{C} 4-5$ to $\mathrm{C} 6-7$ level but the thoracic MRIs were normal. The L1 vertebra showed a compression fracture, with the proximal end of the posterior vertebral rim projection into the spinal canal and impinging on the cord anteriorly (Figure 3a). Consequently, she underwent a posterior cord decompression and transpedicular screw arrangement between T12 and L2 (Figure 3b). Treatment improved the strength of the left lower leg muscles and the gait was unaided 13 months postoperatively.

\section{Discussion}

The complex neuroanatomy of the thoracolumbar junctional area makes interpretation of the features of the syndrome of the epiconus difficult in some patients. Anatomically, the epiconus comprises the cord segment between L4 and $\mathrm{S} 1$, corresponding to the T12 and L1 vertebrae. ${ }^{5}$ The conus medullaris consists of the cord segment between S2 and S5 as well as coccygeal segments. ${ }^{6}$ The lumbosacral nerve roots are collectively termed the cauda equina wihch runs laterally and distally to the epiconus as well as the conus medullaris. It is thus necessary to understand the different levels of neural segments during assessment of patients with neurological symptoms in this region. ${ }^{7,8}$ 
a

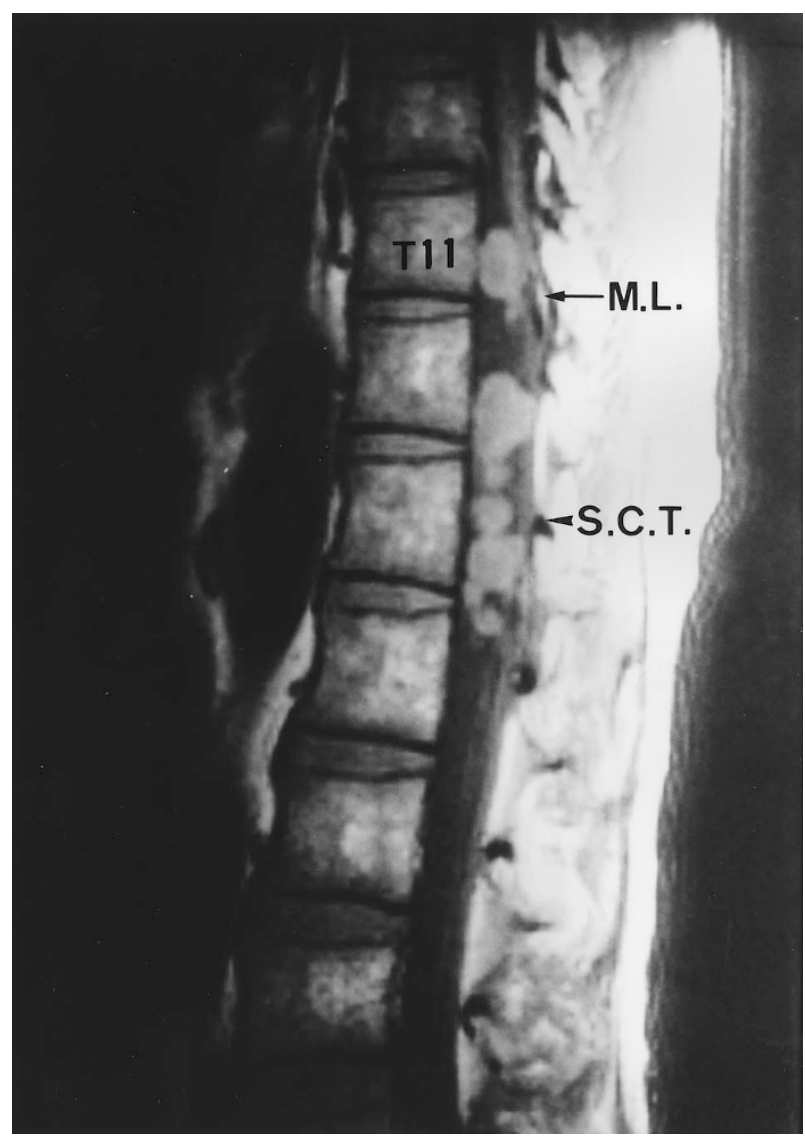

b

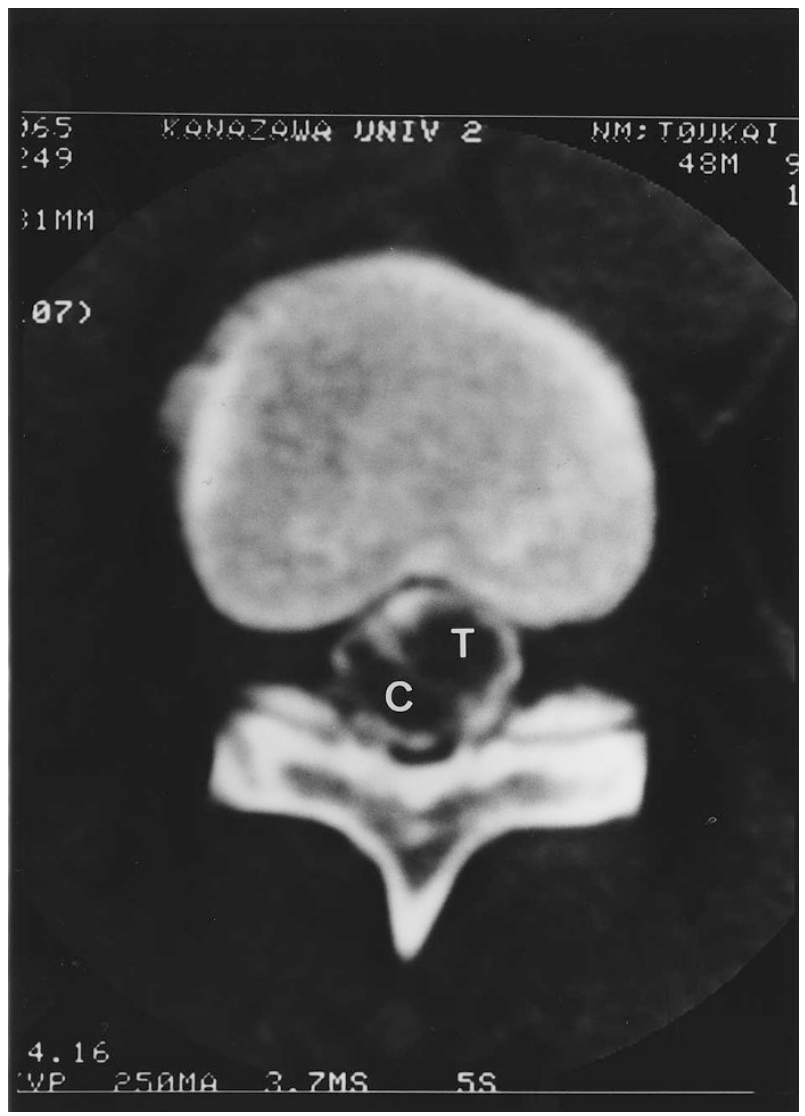

C

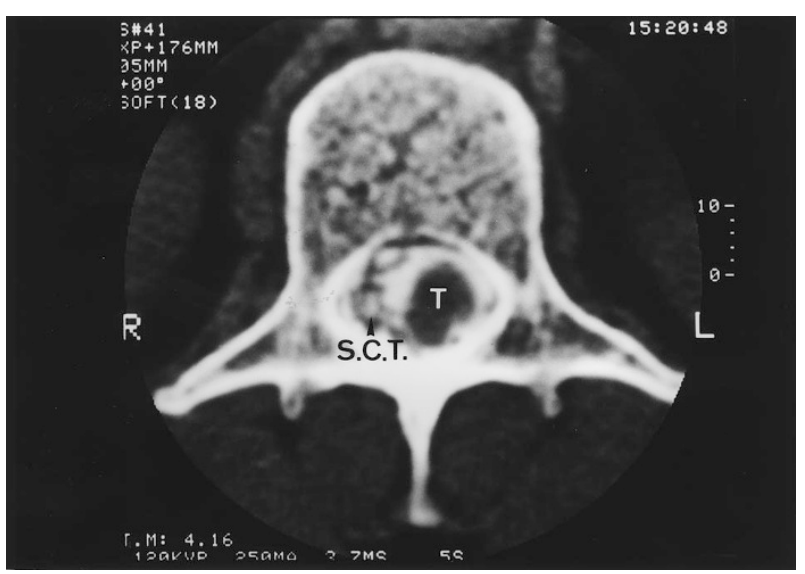

d

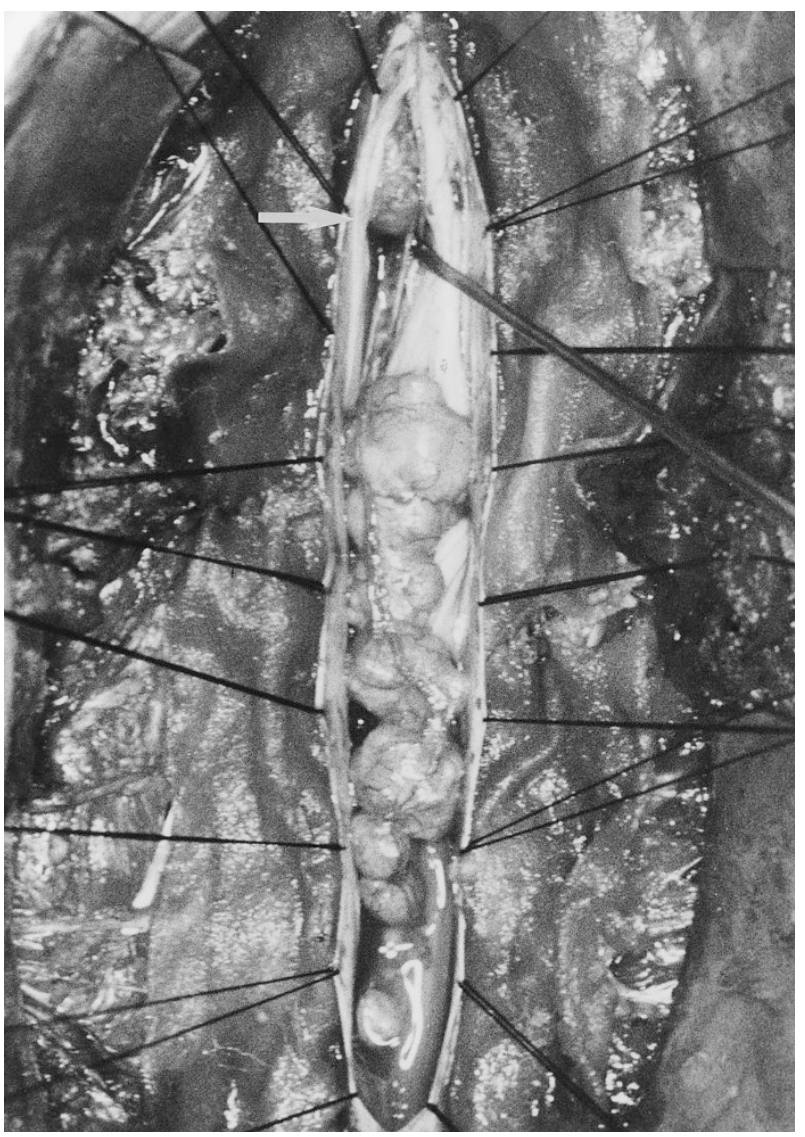

Figure $1 \mathrm{a}-\mathrm{c}$. Neuroimaging and intraoperative photographs of Case 6. a, T1-weighted magnetic resonance imaging (TR, $560 \mathrm{~ms}$; TE, $20 \mathrm{~ms}$ ) (M.L., main lesion; S.C.T., termination of the spinal cord), b, computed tomography myelography at T11 level (T, tumour; $\mathrm{C}$, cord), c, computed tomography myelography at L1 level (T, tumour; S.C.T., termination of the spinal cord). d, intraoperative photograph showing intradural extramedullary neurilemmomas (arrow, tumour at T11-12 level) 
a

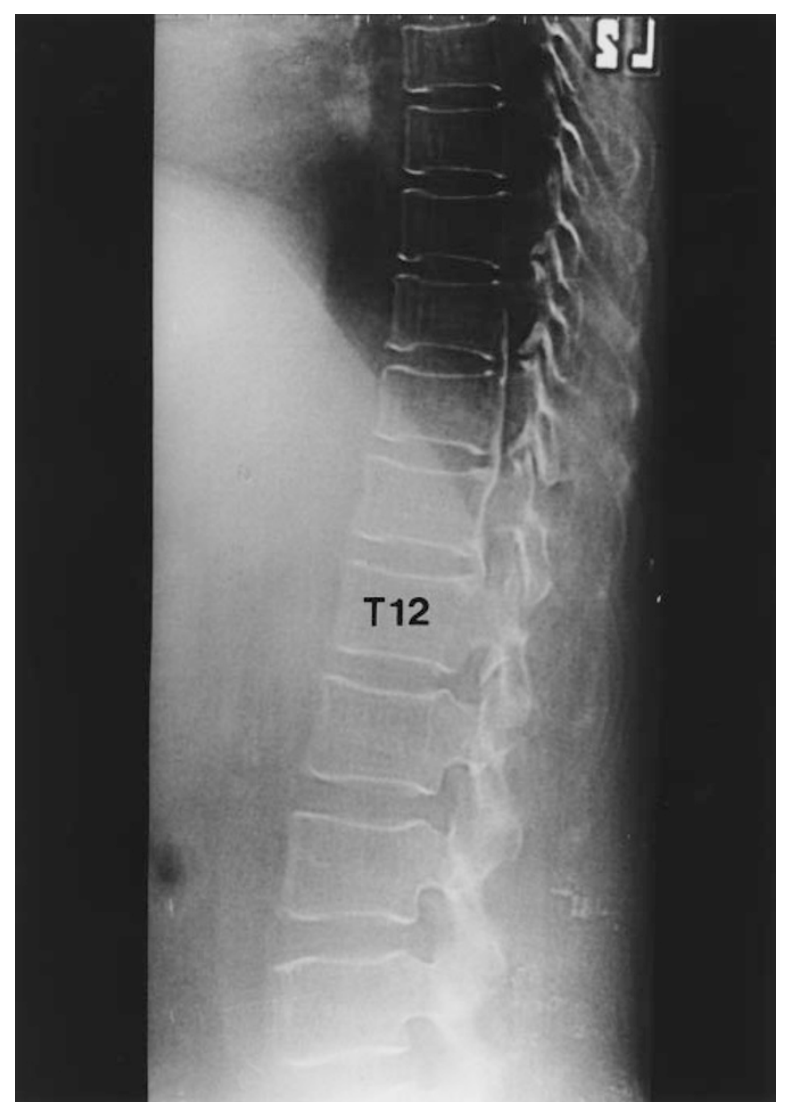

b

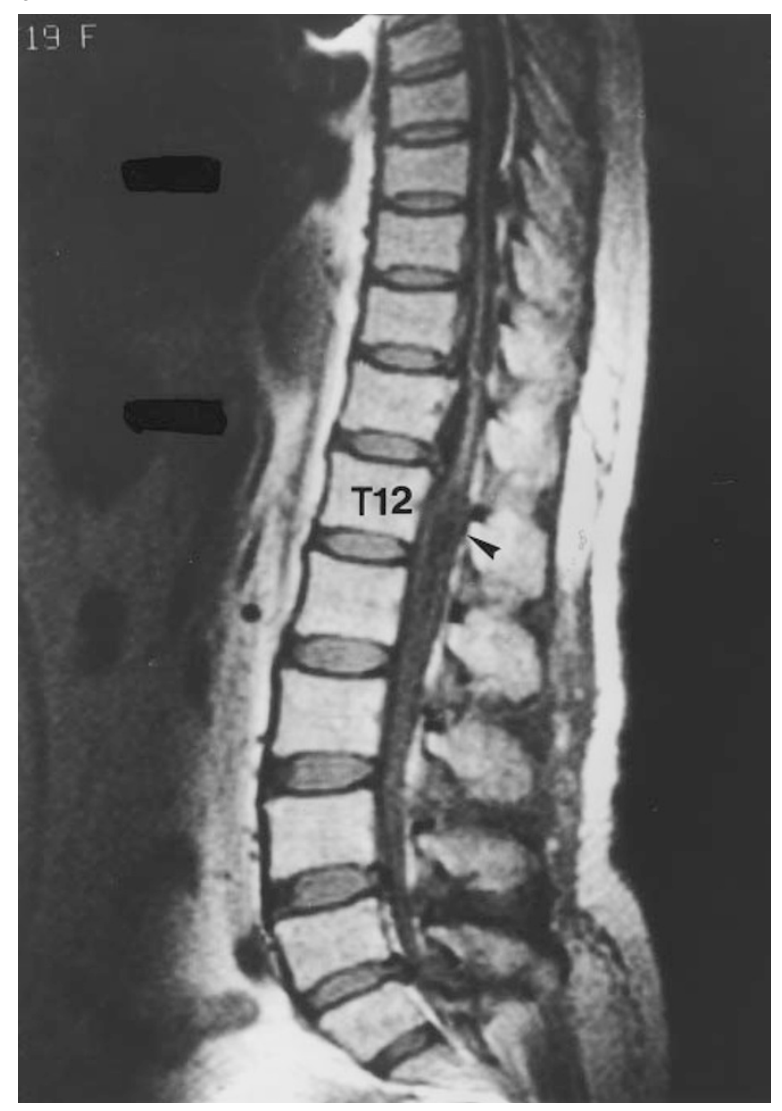

C
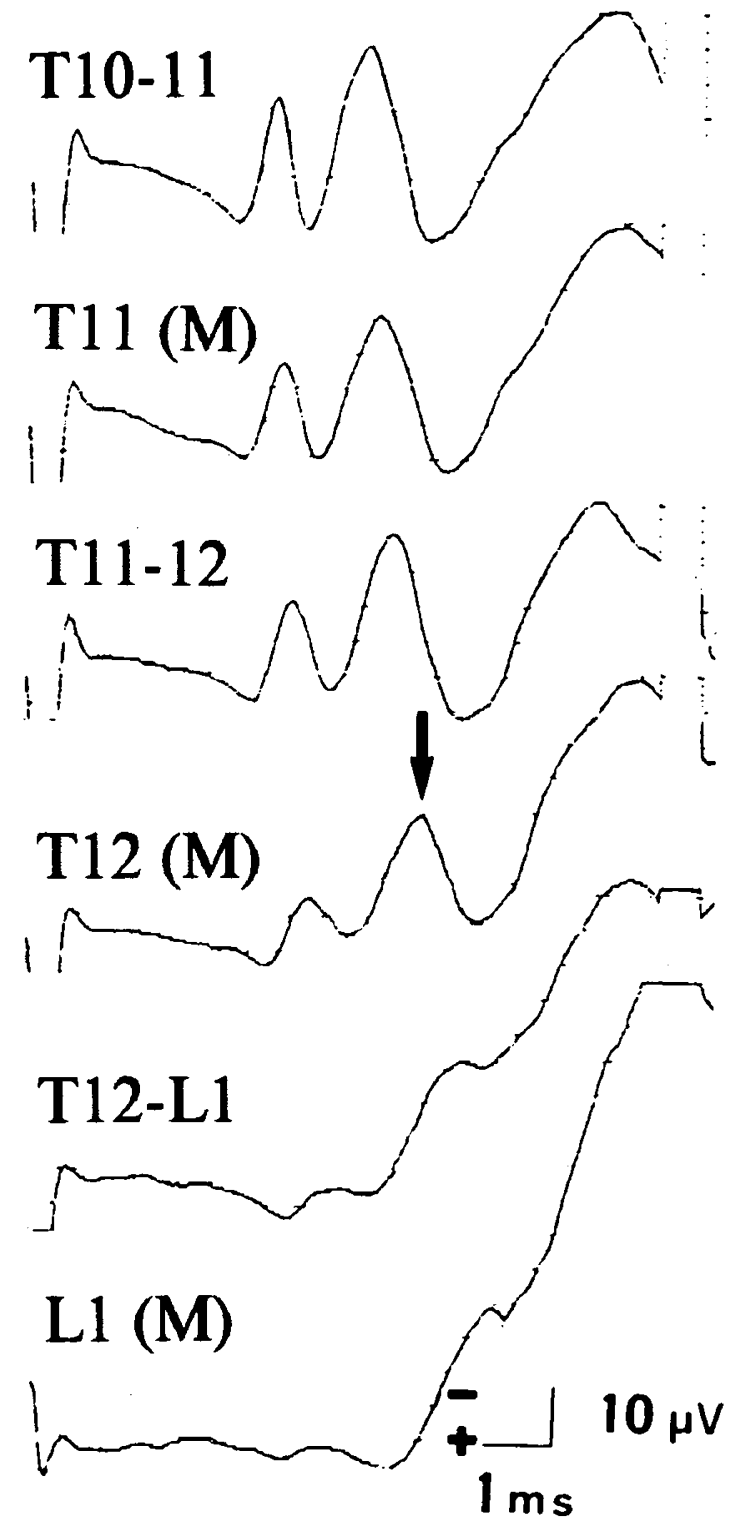

Figure 2 Imaging and epidural spinal cord evoked potentials in Case 10. a, Radiograph showing ossification of the posterior longitudinal ligament at $\mathrm{T} 9, \mathrm{~T} 10$, and ossification of the ligamentum flavum from T8-9 to T11-12. b, T1weighted magnetic resonance image (TR, $560 \mathrm{~ms}$; TE, $20 \mathrm{~ms}$ ) showing an irregular signal intensity within the dural sac (arrow), compressed by the ossified ligamentum flavum at T11-12, c, epidural spinal cord evoked potentials showing a latency delay of the second negative component (arrow), suggestive of a conduction disturbance at this level 
a

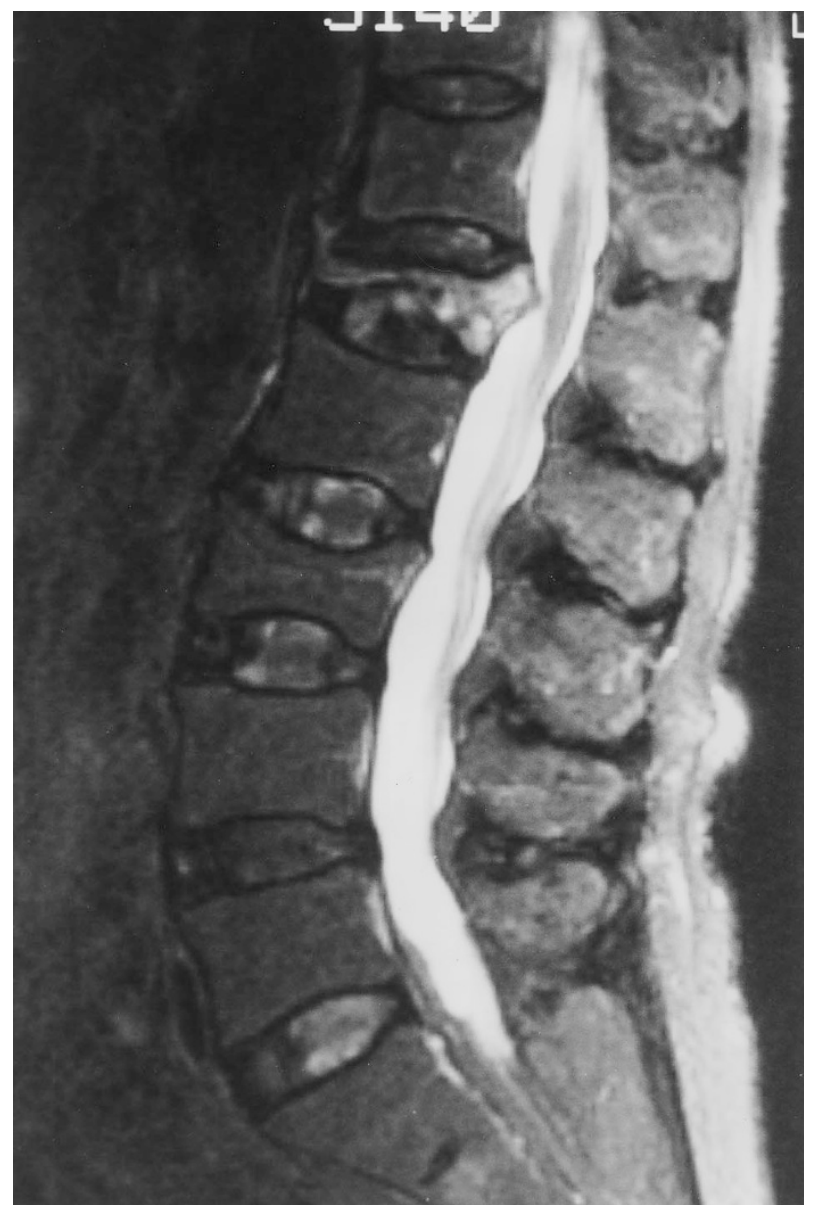

b

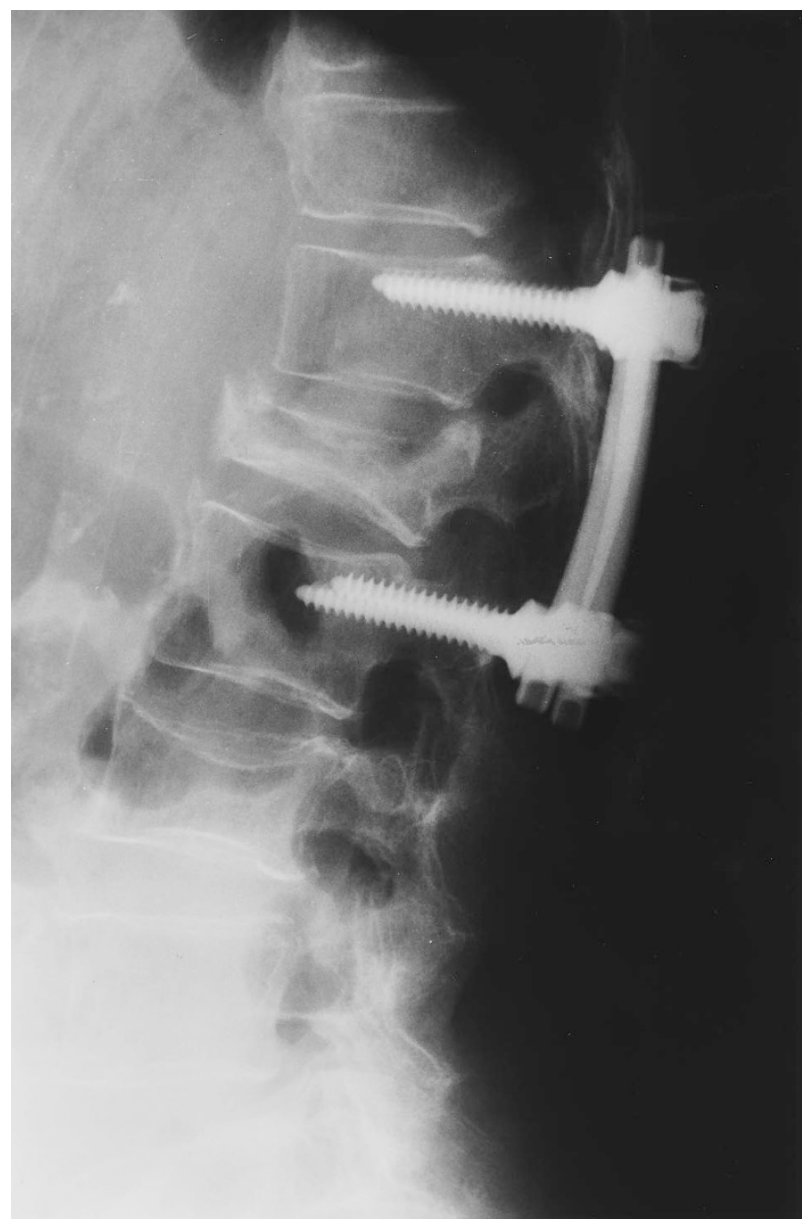

Figure 3 Radiological images in Case 13. a, T2-weighted magnetic resonance imaging (TR, $1900 \mathrm{~ms}$; TE, $200 \mathrm{~ms}$ ) showing cord compression at the rostral end of the collapsed L1 vertebra (L1), b, radiograph after a transpedicular screw arrangement

The epiconus syndrome presents with the following clinical features. ${ }^{1,2,8}$ (1) A sensory disturbance in the leg (transverse, saddle, radicular, or socks type). (2) Motor deficit as a sign of lower motor neuron involvement (foot drop, fasciculation, muscle atrophy). (3) Diminished deep tendon reflexes. (4) Occasional coexistence of positive pathological reflexes (Babinski's and Chaddock's signs). (5) Diminished vibration sensation, and (6) bladder and bowel dysfunction. In the presence of a dysfunctional conus medullaris, sensory disturbance in the perineal area (usually saddle type) and diminished vesicourethral as well as anorectal reflexes are significant findings. On the other hand, no significant abnormalities of voluntary leg movement and in deep tendon reflex activity are observed.

The cauda equina syndrome is a well recognised clinical entity with a flaccid type of paraesis and/or intermittent neurogenic symptoms, and infrequently, parasympathetic disorders with unusual symptoms of penile erection as well as urinary incontinence. Our patients presented here showed primarily the radicular type, although some presented with the socks type, of sensory deficits and a variable degree of muscle atrophy compatible with lower motor neuron compromise, but not caused by nerve root or peripheral nerve damage. Eight patients were initially misdiagnosed as having a lumbar disc herniation and/or idiopathic peroneal nerve palsy. The differential diagnosis should include old poliomyelitis, tethered cord syndrome as well as spinal dysraphism, amyotrophic lateral sclerosis, hereditary spastic paraplegia, hereditary sensorimotor neuropathy (Charcot-MarieTooth; Dejerine-Sottas), and spinal progressive muscular atrophy (Kennedy-Alter-Sung). With these conditions in mind, one should carefully assess the neurological condition in patients presenting with unusual leg muscle atrophy.

Reimann and Anson ${ }^{9}$ and Austin ${ }^{1}$ reported that the spinal cord terminates between L1 and L2 vertebrae. 
Bullough and Boachie-Adjei ${ }^{5}$ also described the spinal cord ending to become the film terminale around the level of L1-2. Based on anatomical observations, Louis ${ }^{10}$ indicated that the spinal cord terminates at T12-L1 disc level in $16 \%$ of the adult European Caucasians, at L1 level in 44\%, at L1-L2 disc level in $20 \%$, at L2 level in 16\%, and at L2-3 disc level in $4 \%$. However, in adult Africans, the level is more caudal at L1-L2 disc level in 52\% and at L2 level in $24 \%$ of the examined population sample. No differences in the vertebral level were observed due to gender or age. Examination of 240 cadavers by Needles ${ }^{11}$ showed that the level of spinal cord termination was between the middle third of T12 and the lower third of L3, while the level was between the lower third of L1 and the upper third of L2 vertebrae in $49 \%$ of cases. He also found that in the African Americans, the spinal cord terminated at a lower level than that of the Caucasians, and the spinal cord ended in females at a lower level than that of males. In the Japanese, Mori ${ }^{12}$ reported that the incidence of spinal cord termination at L1 level was $43 \%$, while in $41 \%$ it terminated at L1L2 disc level and in 7\% at L2 vertebra. Based on the above and other studies, it seems that differences in the level of spinal cord termination are present and some are explained by race and age differences. ${ }^{9,11,13,14}$

It is important that a careful search of the cord lesions should be made in patients with a radiculartype of muscular atrophy as well as sensory deficit in the lower extremity. Such epiconus lesions are usually located several vertebrae proximal to the level of spinal cord termination. Our findings suggested that the termination of the spinal cord was approximately 1.6 vertebrae distal to the level of the epiconus compromise.

\section{References}

1 Austin GM. Tumours of the Lumbosacral Cord and Cauda Equina. In: The Spinal Cord. Igaku-Shoin: Tokyo, New York 1983; pp 292-311.

2 Tashiro K. Symptomatology of thoraco-lumbar junction lesions. Spine Spinal Cord (Tokyo) 1990; 3: 413-420.

3 Baba $\mathrm{H}$ et al. Spinal cord evoked potentials in thoracic myelopathy with multilevel vertebral involvement. Spine 1993; 17: $1291-1295$.

4 Baba $\mathrm{H}$ et al. Spinal cord evoked potential monitoring for cervical and thoracic compressive myelopathy. Paraplegia 1996; 34: $100-106$.

5 Bullough PG, Boachie-Adjei O. Development of the Spinal Cord. In: Atlas of the Spinal Diseases. JB Lippincott: Philadelphia, 1988, pp 53.

6 DiPietro MA. The conus medullaris: normal US findings throughout childhood. Radiology 1993; 188: 149-153.

7 Elsberg CA. The symptoms of Spinal Disease at Different Levels and in Different Regions of the Cord. In: Diagnosis and Treatment of Surgical Diseases of the Spinal Cord and Its Membranes. WB Saunders: Philadelphia, 1916, pp 60-66.

8 Haymaker W. Localization of Lesions Involving Lower Spinal Segments and the Cauda Equina. In: Bing's Local Diagnosis in Neurological Diagnosis. CV Mosby: St Louis, 1969, pp 105-113.

9 Reimann AF, Anson BJ. Vertebral level of termination of the spinal cord with report of a case of a sacral cord. Anat Rec 1944; 88: $127-138$

10 Louis R. Anatomy of the Spinal Cord: A. Termination of the Spinal Cord. In: Surgery of the Spine: Surgical Anatomy and Operative Approaches. Translated to the Japanese Edition by Otani K, Springer-Verlag: Tokyo, 1995, pp 90.

11 Needles JH. The caudal level of termination of the spinal cord in American Whites and American Negroes. Anat Rec 1935; 63: $417-424$.

12 Mori O. Spinal Cord Anatomy. In: Human Anatomy. Kanehara Pub. Co.: Tokyo, 1973, pp 186-199.

13 Barson AJ. The vertebral level of the termination of the spinal cord during normal and abnormal development. J Anat 1969; 106: 489 - 497.

14 Jit I, Charnalia VM. The vertebral level of the termination of the spinal cord. J Anat Soc India 1958; 2: $93-102$. 